\title{
Smoking adversely affects treatment response, outcome and relapse in tuberculosis
}

Chi C. Leung ${ }^{1}$, Wing W. Yew ${ }^{2}$, Chi K. Chan ${ }^{1}$, Kwok C. Chang ${ }^{1}$, Wing S. Law ${ }^{1}$, Shuk N. Lee ${ }^{1}$, Lai B. Tai ${ }^{1}$, Eric C.C. Leung ${ }^{1}$, Ronald K.F. Au ${ }^{1}$, Shan S. Huang ${ }^{1}$ and Cheuk M. Tam ${ }^{1}$

\section{Affiliations:}

${ }^{1}$ TB and Chest Service, Dept of Health, Hong Kong, China.

${ }^{2}$ Stanley Ho Centre for Emerging Infectious Diseases, The Chinese University of Hong Kong, Hong Kong, China.

\section{Correspondence:}

Chi Chiu Leung, Wanchai Chest Clinic, 99 Kennedy Road, Wanchai, Hong Kong, China.

E-mail: cc_leungadh.gov.hk

ABSTRACT The impact of smoking on tuberculosis outcome was evaluated in a territory-wide treatment programme.

16345 consecutive patients undergoing chemotherapy for active tuberculosis in government chest clinics in Hong Kong from 2001 to 2003 were followed up prospectively for 2 years for treatment outcome and subsequently tracked through the territory-wide tuberculosis notification registry for relapse until the end of 2012 .

Smoking was associated with more extensive lung disease, lung cavitation and positive sputum smear and culture at the baseline. In both current smokers and ex-smokers, sputum smears and cultures were significantly more likely to remain positive after 2 months of treatment. Both categories of smokers were significantly less likely to achieve cure or treatment completion within 2 years. Overall, $16.7 \%$ of unsuccessful treatment outcomes were attributable to smoking, with the key contributor being default in current smokers and death in ex-smokers. Among successful treatment completers, there was a clear gradient (hazard ratios of $1.00,1.33$ and 1.63) of relapse risk from never-smokers to ex-smokers and current smokers, with an overall population attributable risk of 19.4\% (current smokers: $12.2 \%$; ex-smokers: $7.2 \%$ ).

Smoking adversely affects baseline disease severity, bacteriological response, treatment outcome and relapse in tuberculosis. Smoking cessation likely reduces relapse and secondary transmission.

@ERSpublications

Smoking adversely affects treatment response in TB. Smoking cessation reduces relapse and secondary transmission. http://ow.ly/CjFZG

For editorial comments see Eur Respir J 2015; 45: 583-585 [DOI: 10.1183/09031936.00221814].

Received: June 232014 | Accepted after revision: Sept 222014 | First published online: Oct 302014

Conflict of interest: None declared.

Copyright OERS 2015 


\section{Introduction}

The association between smoking and tuberculosis (TB) was investigated as early as 1918 [1]. In recent years, active smoking has been shown to be associated with TB after controlling for a series of potential confounders in a number of prospective cohort studies [2-4]. Active smoking has also been associated with TB infection [5, 6] and mortality [7-10], albeit with more heterogeneous effect sizes and/or lesser degrees of certainty [11-13]. However, the full impact of smoking on the TB patients has not been evaluated in sufficient depth, especially in the setting of a national or territorial TB control programme.

In Hong Kong, the annual TB notification rate remains high at $\sim 70$ per 100000 in recent years [14]. 18 government chest clinics offer free programmatic case-finding and treatment services for TB patients under a centralised TB and Chest Service of the Dept of Health, with estimated programme coverage of over $80 \%$. Standard short-course regimens are used in line with the recommendations of the World Health Organization (WHO). Baseline smoking status is regularly captured at initiation of TB treatment. Brief advice on smoking cessation is given during health education, and referral for further nonpharmacological and/or pharmacological interventions is made, as deemed appropriate. Patients are regularly followed up for 2 years after initiation of TB treatment to facilitate cohort analysis of treatment outcome. A statutory TB notification system is in place, whereby medical practitioners are required to notify all diagnosed TB cases to the Dept of Health. With the availability of appropriate service infrastructure, a prospective cohort study was, therefore, conducted to examine the impact of smoking on disease severity, bacteriological response, treatment outcome and relapse among consecutive patients managed under the centralised TB programme.

\section{Materials and methods}

Consecutive patients who underwent chemotherapy for active TB at the 18 clinics of the Hong Kong TB and Chest Service from January 1, 2001 to December 31, 2003 were followed up prospectively in clinics to assess treatment progress and outcome for up to 2 years. Those who successfully completed treatment were then tracked by linking with the territory-wide TB registry and death registry using identity card number as the unique identifier for relapse, death or until December 31,2012, whichever was the earliest. Patients who were not local residents (and, thus, without a valid local identity card number) were excluded to minimise loss to follow-up through population movement. Patients whose subsequent culture isolates showed drug resistance to either isoniazid and/or rifampicin were also excluded because of their small numbers and potential confounding effects. The diagnosis and clinical information of all identified relapse TB cases were verified by reviewing the medical records retrieved from the chest clinics and other relevant sources.

An ever-smoker was defined as one who had smoked the equivalent of at least one cigarette a day for a period of 1 year. An ex-smoker was defined as an ever-smoker who had stopped smoking for at least 1 year before the current TB episode, and a current smoker as an ever-smoker who was still smoking or had stopped smoking for less than 1 year. Patients who did not fulfil the criterion of an ever-smoker were classified as never-smokers. Relapse was defined as recurrence of TB after successful completion of treatment, either proven by isolation of Mycobacterium tuberculosis, or in the absence of bacteriological confirmation, recurrence diagnosed on clinical, radiological and/or histological grounds together with an appropriate response to treatment.

The clinical parameters were compared by smoking status at the baseline. For those with positive sputum smears or cultures, the bacteriological response was assessed by smear and/or culture conversion at 2 months after initiation of treatment. The proportion of patients who successfully completed treatment (cure or treatment completion) within 24 months were then compared by baseline smoking status, with control of the potentially confounding baseline parameters of sex, age, ethnicity, residency status, employment status, housing situation, alcohol dependence, drug abuse, diabetes mellitus, HIV status, retreatment versus new case, extent of lung involvement, lung cavity, and sputum status. For successful treatment completers, the effect of smoking on subsequent relapse was assessed, with adjustment for other baseline characteristics.

Chi-squared was used for categorical variables and ANOVA was used for numerical variables in univariable analysis. Logistic regression analysis was used for multivariable analysis of treatment outcome within an uncensored time-interval of 2 years. Kaplan-Meier analysis was used for univariable analysis of relapse and Cox proportional hazards modelling was used in multivariable analysis to adjust for potential confounding baseline parameters, using the backward conditional approach with probability to retain being 0.05 and probability to remove being 0.10 . The proportional hazard assumption of the Cox model was assessed by inspection of the $\log$ minus $\log$ curve. A two-tailed p-value of $<0.05$ was taken as statistically significant. 
The population attributable risk was estimated by a modified version of the Levin's formula as follows $[2,15]$ :

Population attributable risk $=($ observed rate - unexposed rate $) /$ observed rate

$$
\begin{aligned}
& =1-(\text { unexposed rate/observed rate }) \\
& =1-\left(\left(\mathrm{N}_{1}+\mathrm{N}_{2}+\mathrm{N}_{3}\right) \times 1 /\left(\mathrm{N}_{1} \mathrm{HR}_{13}+\mathrm{N}_{2} \mathrm{HR}_{23}+\mathrm{N}_{3} \times 1\right)\right)
\end{aligned}
$$

where $\mathrm{N}_{1}, \mathrm{~N}_{2}$ and $\mathrm{N}_{3}$ were the number of current smokers, ex-smokers and never-smokers, respectively, and $\mathrm{HR}_{13}$ and $\mathrm{HR}_{23}$ were the adjusted hazard ratios of current smokers versus never-smokers and ex-smokers versus never-smokers, respectively. Never-smokers were used as the reference group with a hazard ratio of 1 .

The study was approved by the Ethics Committee of the Dept of Health of Hong Kong (Hong Kong, China).

\section{Results}

Out of 17415 consecutive TB patients, 212 (1.2\%) with subsequently revised diagnosis, 540 (3.1\%) with isoniazid or rifampicin resistance and $318(1.8 \%)$ with unknown smoking status at initiation of treatment were excluded, leaving 16345 patients for analysis. Table 1 summarises their baseline characteristics by smoking status. Smoking was significantly associated not only with sociodemographic variables but also with history of previous TB treatment, extent of lung disease, lung cavity, and positive sputum bacteriology at the baseline.

Table 2 shows the bacteriological status at 2 months among patients with positive bacteriology at the baseline. Both ex-smokers and current smokers were significantly more likely to have persistently positive sputum smear and culture after 2 months of treatment, and a clear gradient was seen in increasing rates of positive sputum culture from never-smokers to ex-smokers and current smokers.

Table 3 summarises the treatment outcomes at 24 months after initiation of treatment. Among the cohort, a total of 13349 (81.7\%) patients were successfully treated (cure or treatment completion) within 2 years. The major contributor to unsuccessful treatment outcome (outcome other than cure or treatment completion) was default among current smokers, while death was key reason for the poorer outcome among ex-smokers. Table 4 summarises the relationship between smoking status and treatment success in both univariable analysis and multivariable analysis. On applying the modified Levin formula to the reciprocals of the adjusted odds ratios for treatment success (equivalent to the adjusted odds ratios for

\begin{tabular}{|c|c|c|c|c|}
\hline & \multicolumn{3}{|c|}{ Smoking status } & \multirow[t]{2}{*}{ p-value } \\
\hline & Never-smokers & Ex-smokers & Current smokers & \\
\hline Subjects $\mathrm{n}$ & 7687 & 4708 & 3950 & \\
\hline Mean $\pm s D$ age years & $47.0 \pm 21.7$ & $63.8 \pm 17.4$ & $49.9 \pm 16.8$ & $<0.001$ \\
\hline Chinese & 91.4 & 98.3 & 97.6 & $<0.001$ \\
\hline Permanent residents & 89.3 & 98.0 & 97.5 & $<0.001$ \\
\hline Alcohol dependence & 0.4 & 1.4 & 4.4 & $<0.001$ \\
\hline Drug abuse & 0.1 & 0.7 & 4.1 & $<0.001$ \\
\hline Diabetes mellitus & 11.4 & 17.3 & 9.2 & $<0.001$ \\
\hline HIV & 0.3 & 0.5 & 0.6 & 0.034 \\
\hline Retreatment & 9.3 & 18.3 & 14.0 & $<0.001$ \\
\hline Lung involvement & & & & $<0.001$ \\
\hline Sputum status & & & & $<0.001$ \\
\hline Smear and culture negative & 45.0 & 29.7 & 34.5 & \\
\hline Culture positive only & 25.8 & 30.8 & 28.8 & \\
\hline Smear positive & 29.2 & 39.6 & 36.7 & \\
\hline
\end{tabular}

TABLE 1 Baseline characteristics of the cohort by smoking status

Data are presented as \%, unless otherwise stated. " : street sleeper, institutional clients, or living in bed spaces or cubicles; " total area of observable lung lesions as compared with that of the right upper lobe. 
TABLE 2 Smear and culture conversion at the end of 2 months

\begin{tabular}{lcccc} 
& \multicolumn{3}{c}{ Baseline smoking status } & p-value \\
\cline { 2 - 4 } & Never-smokers & Ex-smokers & Current smokers & \\
\hline Smear & & & & $<0.001$ \\
Subjects n & 2246 & 1864 & 1450 & \\
Not available & 26.5 & 26.7 & 22.1 & \\
Converted & 67.7 & 62.3 & 64.7 & $<0.001$ \\
$\quad$ Not converted & 5.8 & 11.1 & 9.4 & \\
Culture & & & 2473 & \\
Subjects n & 3919 & 3102 & 26.4 & \\
Not available & 31.0 & 30.7 & 64.1 & \\
Converted & 64.1 & 62.2 & 9.4 & \\
Not converted & 4.9 & 7.2 & & \\
\hline
\end{tabular}

Data are presented as $\%$, unless otherwise stated.

unsuccessful treatment outcome) with respective baseline smoking status, $16.7 \%$ (95\% CI 10.4-22.5\%) of unsuccessful treatment outcome was attributable to smoking.

Among the 13349 patients who were successfully treated, 426 relapses were detected after 107686 person-years of post-treatment follow-up, at a rate of 396 per 100000 person-years. Of these relapses, 349 (81.9\%) involved the lung alone, 66 (15.5\%) involved extrapulmonary sites alone and 11 (2.6\%) involved both. Overall, $204(47.8 \%)$ of these relapses were bacteriologically confirmed. Table 5 summarises the results of univariable and multivariable survival analyses of the effect of baseline smoking status on $\mathrm{TB}$ relapse. A clear gradient of increasing risk of relapse was observed from never-smokers to ex-smokers and current smokers in both analyses. Consistent results were also obtained in sensitivity analyses with respect to bacteriologically confirmed relapses. Figure 1 shows the cumulative hazard curves by baseline smoking status in Cox proportional hazards modelling using the backward conditional approach. On applying the modified Levin formula to the adjusted hazard ratios for TB relapse with respective baseline smoking status, $19.4 \%$ (95\% CI 7.5-30.9\%) of $\mathrm{TB}$ relapse among the successful treatment completers was attributable to smoking, with current smoking accounting for $12.2 \%$ (95\% CI 6.5-17.7\%) and ex-smoking accounting for $7.2 \%$ (95\% CI $1.0-13.3 \%)$.

\section{Discussion}

In this study, both current smokers and ex-smokers were associated with more extensive lung disease, lung cavitation and positive sputum bacteriology at baseline (table 1) and increased risks of persistently positive smear and culture after 2 months of treatment as compared with never-smokers (table 2). While both categories of smokers were less likely to achieve cure or treatment completion within 2 years in both univariable and multivariable analysis (table 3 and 4), the key contributor for the poorer treatment outcome was default in current smokers and death in ex-smokers (table 3). Among successful treatment completers, there was a clear gradient (hazard ratio: 1.00, 1.33 and 1.63, respectively) of relapse risk from never-smokers to ex-smokers and current smokers.

TABLE 3 Treatment outcomes measured at 24 months after initiation of treatment

\begin{tabular}{lcccc} 
& \multicolumn{3}{c}{ Smoking status } & p-value \\
\cline { 2 - 4 } & Never-smokers & Ex-smokers & Current smokers & \\
\hline Treatment success & $6517(84.7)$ & $3611(76.7)$ & $3221(81.5)$ & $<0.001$ \\
Still on treatment & $17(0.2)$ & $19(0.4)$ & $16(0.4)$ & \\
Transfer out & $279(3.6)$ & $95(2.0)$ & $80(2.0)$ & \\
Defaulted & $531(6.9)$ & $374(7.9)$ & $176(11.6)$ & \\
Death lall causes) & $343(4.5)$ & $609(12.9)$ & $3950(100)$ & \\
Subtotal & $7687(100)$ & $4708(100)$ &
\end{tabular}

Data are presented as $\mathrm{n}(\%)$, unless otherwise stated. \#: cure or treatment completion (successfully completed treatment of $\geqslant 6$ months for new cases and $\geqslant 8$ months for retreatment cases), irrespective of subsequent relapse or death or loss to follow-up. 
TABLE 4 Univariable analysis and multiple logistic regression analysis on successful treatment completion by baseline smoking status

\begin{tabular}{|c|c|c|c|c|c|c|}
\hline & Subjects $n$ & Treatment success $\%$ & OR $(95 \% \mathrm{CI})$ & p-value & Adjusted OR $(95 \% \mathrm{CI})^{\pi}$ & p-value \\
\hline Never-smokers & 7687 & 84.8 & Reference & & Reference & \\
\hline Current smokers & 3950 & 81.5 & $0.79(0.72-0.88)$ & & $0.72(0.64-0.82)$ & \\
\hline Overall & 16345 & 81.7 & & & & \\
\hline
\end{tabular}

\#: cure or treatment completion (successfully completed treatment of $\geqslant 6$ months for new cases and $\geqslant 8$ months for retreatment cases), irrespective of subsequent relapse or death or loss to follow-up. ๆ: adjusted for all potential confounding variables as listed in table 1, with all variables forced into the model; age was included as a continuous variable because of its reverse linear association with treatment success.

Our observations of more extensive lung disease, lung cavitation and positive sputum smears among current smokers and ex-smokers corroborate those reported in previous studies [16-18] and point to potentially enhanced secondary transmission risk [19]. In this study, both current smokers and ex-smokers were 1.5-2 times as likely to remain smear-positive and culture-positive after 2 months of treatment, closely parallel to the observation in a previous Brazilian case-control study that ever-smokers who smoked $>20$ cigarettes per day were also reported to be two-fold as likely to remain culture-positive after 2 months of treatment [20]. The slower bacteriological response raises concern over persistent transmission risk after the initiation of treatment, even for patients with initially fully drug-sensitive TB as included in the current study.

Similar to previous studies $[21,22]$, smoking was negatively associated with cure or treatment completion in this study, even after controlling for baseline sociodemographic variables, comorbidities, extent of lung disease, lung cavitation and bacteriology. As expected for the use of standard four-drug short course regimens for drug-susceptible $\mathrm{TB}$, under a fully functioning treatment programme setting that also allowed individualised regimen modification and treatment prolongation, treatment failure was not an expected event. However, the default rate was as high as $11.6 \%$ among current smokers, which is double that in never-smokers. This finding further substantiates the significant association between smoking and treatment default as reported in previous studies [23, 24]. A major difference in mean age was observed between ex-smokers and current smokers. The high mortality rate among ex-smokers probably reflected the combined effects of smoking [7-10] and age-related comorbidities [25, 26]. It also remained a possibility that some of these comorbidities could have promoted the motivation for smoking cessation in the first place, and then increased the higher mortality on later follow-up.

While the independent association between smoking and TB relapse in this study corroborates those reported in previous smaller scale studies [27-29], a clear gradient of effect was seen from never-smokers to ex-smokers (adjusted hazard ratio: 1.3) and current smokers (adjusted hazard ratio: 1.6), and these

TABLE 5 Univariable and multivariable of risk of tuberculosis relapse by baseline smoking status

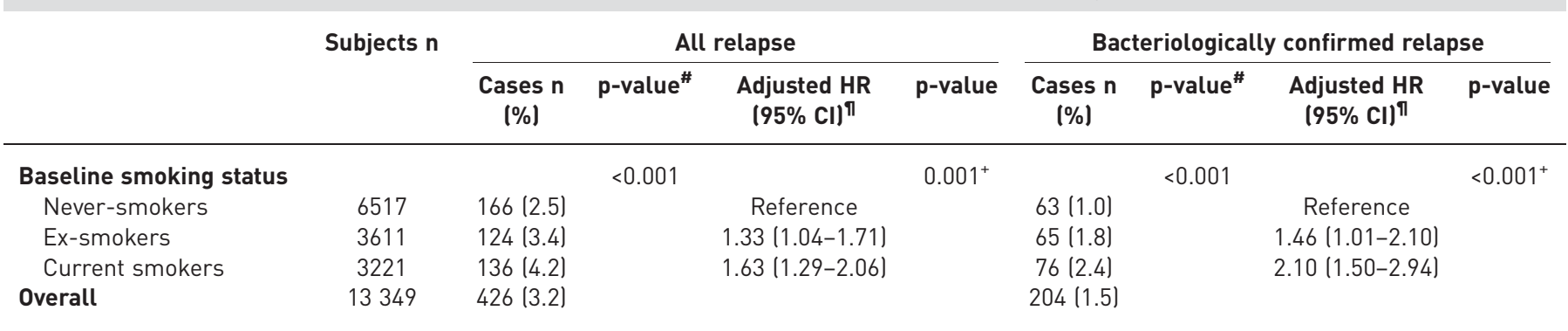

HR: hazard ratio. "\#: Kaplan-Meier analysis, log-rank test. ": adjusted for all variables listed in table 1, using the backward conditional approach with probability to retain being 0.05 and probability to remove being 0.10 ; age was included as a continuous variable because of its linear association with relapse; besides baseline smoking status, only age, retreatment case, extent of lung involvement and baseline sputum status were retained in the final model. ${ }^{+}: \mathrm{p}$-value for trend across categories of never-smokers, ex-smokers and current smokers $<0.001$. 
FIGURE 1 Cumulative hazards for tuberculosis relapse by smoking status in Cox proportional hazards analysis.

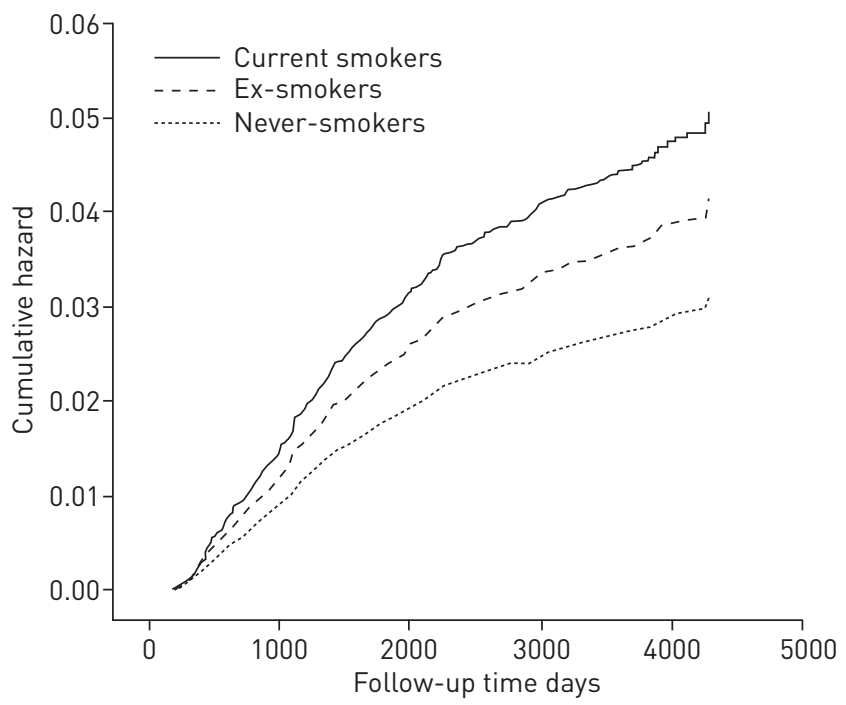

effect sizes were substantially lower than the two- to three-fold risks reported in the previous studies. Our observed effect sizes closely paralleled the estimated effect size of 1.5 for the effect of smoking on progression from latent TB infection to active disease (overall effect on disease/effect on infection) [10, 30] and the differences from previous observations could be attributable to our adjustment for baseline disease severity in addition to other potential confounding factors.

The effects of smoking on baseline disease parameters (lung cavitation, positive sputum smear and culture), and slower smear and culture conversion after initiation of treatment highlight a critical need for prevention of nosocomial transmission. Even for patients with initially drug-sensitive TB, the treatment completion rates fell substantially below the WHO target of 85\% [31] among both current smokers and ex-smokers in this study. The high percentage of current smokers who default treatment also raises concern over the emergence of drug resistance and secondary spread within the community. Even among those who were successfully treated, they still ran a substantially higher risk (396 per 100000 person-years) for developing active TB again than overall TB risk (70 per 100000 person-years) in the general population [14]. Smoking contributed substantially to such relapses, with a major part of the excess risk occurring among those who continued to smoke. Passive smoking has also been shown to increase the risk of both TB infection [32] and disease [33]. Patients who continue to smoke, therefore, pose a risk not only to themselves, but also to every other person simultaneously exposed to their infection and cigarette smoke. The situation is expected to be even worse for drug-resistant TB as the effect of smoking is probably compounded by the generally poorer response to available drugs. Smoking cessation is, therefore, called for in all TB patients who smoke to protect everyone from healthcare facilities to the general community.

The number of cigarettes smoked per day or the duration of smoking was not regularly captured in this study, thus preventing the establishment of a dose-response relationship. However, the considerable size of our cohort helped to overcome the power limitations of previous studies, especially in discerning the differential effects of current smoking and previous smoking. Data capture was also facilitated by the well-developed health infrastructure with a freely accessible TB service and regular follow-up of TB patients, as well as the statutory TB notification system and death registration using the identity card number as the common unique identifier. In addition to patients with only extrapulmonary disease, quite a large number of patients failed to produce sputum spontaneously for examination at 2 months with improvement in their clinical condition, and sputum induction was not normally performed for disease monitoring in the regular service setting. While incomplete case ascertainment or misclassification could occur as in other similar studies, good validity is still expected for internal comparison among different subcategories of smokers within the same cohort. Only $47.8 \%$ of $\mathrm{TB}$ relapses were bacteriologically confirmed in this study. However, as shown in the first smear-active study in Hong Kong [34], joint decision by experienced physicians in clinical meetings was useful in achieving a high standard in active disease categorisation. Consistent results were also obtained on restricting the analyses to bacteriologically confirmed relapses. As data on change of smoking status was not systematically captured during treatment, we were not able to assess the impact of smoking cessation during treatment on treatment outcome. However, a cause-effect relationship might be difficult to delineate in the absence of a clear time sequence 
between change in smoking status and treatment effect. Treatment adherence and other causes of loss to follow-up could also confound any observed relationship. The introduction of brief tobacco cessation advice for TB patients has been shown to be feasible in a programme setting [35]. A recent randomised controlled trial in Africa also demonstrated that motivational interviewing by lay counsellors doubled sustained smoking abstinence for at least 6 months among TB patients compared with brief advice alone [36]. A randomised controlled trial assessing the effect of smoking cessation on TB treatment outcome under a programme setting would be worth exploring to definitively answer this question.

\section{References}

1 Webb GB. The effect of the inhalation of cigarette smoke on the lungs: a clinical study. Am Rev Tuberc 1918; March: 25-27.

2 Leung CC, Li T, Lam TH, et al. Smoking and tuberculosis among the elderly in Hong Kong. Am J Respir Crit Care Med 2004; 170: 1027-1033.

3 Leung CC, Yew WW, Law WS, et al. Smoking and tuberculosis among silicotic patients. Eur Respir J 2007; 29: 745-750.

4 Lin HH, Ezzati M, Chang HY, et al. Association between tobacco smoking and active tuberculosis in Taiwan: prospective cohort study. Am J Respir Crit Care Med 2009; 180: 475-480.

5 den Boon S, van Lill SW, Borgdorff MW, et al. Association between smoking and tuberculosis infection: a population survey in a high tuberculosis incidence area. Thorax 2005; 60: 555-557.

6 Anderson RH, Sy FS, Thompson S, et al. Cigarette smoking and tuberculin skin test conversion among incarcerated adults. Am J Prev Med 1997; 13: 175-181.

7 Gajalakshmi V, Peto R, Kanaka TS, et al. Smoking and mortality from tuberculosis and other diseases in India: retrospective study of 43000 adult male deaths and 35000 controls. Lancet 2003; 362: 507-515.

8 Jha P, Jacob B, Gajalakshmi V, et al. A nationally representative case-control study of smoking and death in India. N Engl J Med 2008; 358: 1137-1147.

9 Liu $\mathrm{BQ}$, Peto R, Chen ZM, et al. Emerging tobacco hazards in China: 1. Retrospective proportional mortality study of one million deaths. BMJ 1998; 317: 1411-1422.

10 Lam TH, Ho SY, Hedley AJ, et al. Mortality and smoking in Hong Kong: case-control study of all adult deaths in 1998. BMJ 2001; 323: 361.

11 Bates MN, Khalakdina A, Pai M, et al. Risk of tuberculosis from exposure to tobacco smoke: a systematic review and meta-analysis. Arch Intern Med 2007; 167: 335-342.

12 Slama K, Chiang CY, Enarson DA, et al. Tobacco and tuberculosis: a qualitative systematic review and meta-analysis. Int J Tuberc Lung Dis 2007; 11: 1049-1061.

13 Lin HH, Ezzati M, Murray M. Tobacco smoke, indoor air pollution and tuberculosis: a systematic review and meta-analysis. PLoS Med 2007; 4: e20.

14 Tuberculosis and Chestservice. Annual report of Tuberculosis and Chest Service 2011. Hong Kong, Dept of Health, 2013; p. 10, Appendix 1.

15 Mausner JS, Kramer S. Epidemiology: an Introductory Text. 2nd Edn. Philadelphia, WB Saunders, 1985; p. 173.

16 Leung CC, Yew WW, Chan CK, et al. Smoking and tuberculosis in Hong Kong. Int J Tuberc Lung Dis 2003; 7: 980-986.

17 Altet-Gômez MN, Alcaide J, Godoy P, et al. Clinical and epidemiological aspects of smoking and tuberculosis: a study of 13,038 cases. Int J Tuberc Lung Dis 2005; 9: 430-436.

18 Wang JY, Hsueh PR, Jan IS, et al. The effect of smoking on tuberculosis: different patterns and poorer outcomes. Int J Tuberc Lung Dis 2007; 11: 143-149.

19 Lee MS, Leung CC, Kam KM, et al. Early and late tuberculosis risks among close contacts in Hong Kong. Int J Tuberc Lung Dis 2008; 12: 281-287.

20 Maciel EL, Brioschi AP, Peres RL, et al. Smoking and 2-month culture conversion during anti-tuberculosis treatment. Int J Tuberc Lung Dis 2013; 17: 225-228.

21 Chiang YC, Lin YM, Lee JA, et al. Tobacco consumption is a reversible risk factor associated with reduced successful treatment outcomes of anti-tuberculosis therapy. Int J Infect Dis 2012; 16: e130-e135.

22 Tachfouti N, Nejjari C, Benjelloun MC, et al. Association between smoking status, other factors and tuberculosis treatment failure in Morocco. Int J Tuberc Lung Dis 2011; 15: 838-843.

23 Chang KC, Leung CC, Tam CM. Risk factors for defaulting from anti-tuberculosis treatment under directly observed treatment in Hong Kong. Int J Tuberc Lung Dis 2004; 8: 1492-1498.

24 Kittikraisak W, Burapat C, Kaewsa-ard S, et al. Factors associated with tuberculosis treatment default among HIV-infected tuberculosis patients in Thailand. Trans R Soc Trop Med Hyg 2009; 103: 59-66.

25 Leung CC, Yew WW, Chan CK, et al. Tuberculosis in older people: a retrospective and comparative study from Hong Kong. J Am Geriatr Soc 2002; 50: 1219-1226.

26 Reed GW, Choi H, Lee SY, et al. Impact of diabetes and smoking on mortality in tuberculosis. PLoS One 2013; 8: e58044.

27 d'Arc Lyra Batista J, de Fátima Pessoa Militão de Albuquerque M, de Alencar Ximenes RA, et al. Smoking increases the risk of relapse after successful tuberculosis treatment. Int J Epidemiol 2008; 37: 841-851.

28 Thomas A, Gopi PG, Santha T, et al. Predictors of relapse among pulmonary tuberculosis patients treated in a DOTS programme in South India. Int J Tuberc Lung Dis 2005; 9: 556-561.

29 Yen YF, Yen MY, Lin YS, et al. Smoking increases risk of recurrence after successful anti-tuberculosis treatment: a population-based study. Int J Tuberc Lung Dis 2014; 18: 492-498.

30 Lin HH, Murray M, Cohen T, et al. Effects of smoking and solid-fuel use on COPD, lung cancer, and tuberculosis in China: a time-based, multiple risk factor, modelling study. Lancet 2008; 372: 1473-1483.

31 World Health Organization. Global tuberculosis report 2013. Geneva, World Health Organization, 2013; p. ix and p. 2-3.

32 den Boon S, Verver S, Marais BJ, et al. Association between passive smoking and infection with Mycobacterium tuberculosis in children. Pediatrics 2007; 119: 734-739. 
33 Leung CC, Lam TH, Ho KS, et al. Passive smoking and tuberculosis. Arch Intern Med 2010; 170: 287-292.

34 Hong Kong Chest Service, Tuberculosis Research Centre, Madras, British Medical Research Council. A study of the characteristics and course of sputum smear-negative pulmonary tuberculosis. Tubercle 1981; 62: 155-167.

35 El Sony A, Slama K, Salieh M, et al. Feasibility of brief tobacco cessation advice for tuberculosis patients: a study from Sudan. Int J Tuberc Lung Dis 2007; 11: 150-155.

36 Louwagie GM, Okuyemi KS, Ayo-Yusuf OA. Efficacy of brief motivational interviewing on smoking cessation at tuberculosis clinics in Tshwane, South Africa: a randomized controlled trial. Addiction 2014; 109: 1942-1952. 